\title{
Estrategias pedagógicas que favorecen el aprendizaje de niñas y niños de 0 a 6 años de edad en Villavicencio- Colombia ${ }^{1}$
}

\author{
Alexandra Cortés ${ }^{2}$ \\ Giovanna Garcia ${ }^{3}$
}

Recibido: 16-03-2017

Aceptado: 09-05-2017

\section{RESUMEN}

La educación en los primeros años vida de es un proceso continuo e integral, producto de múltiples experiencias que brinda la escuela a los niños y niñas, a través de la interacción con la sociedad y con el medio ambiente, siendo la base de la formación integral del ser humano. Unas de las tantas herramientas o estrategias pedagógicas son el juego, las artes escénicas, la pintura y la lectura, las cuales se transforman en estrategias lúdico-pedagógicas que favorecen el desarrollo integral del niño. Esta revisión bibliográfica tiene como objetivo presentar los resultados de diversas investigaciones que contienen pluralidad de herramientas metodológicas, que los docentes pueden emplear en el aula de clase, para desarrollar en el niño un mejor aprendizaje. Se encuentra que existe una variedad de estrategias implementadas por los maestros en las instituciones educativas; sin embargo, no se encuentra una investigación que produzca e integre las diferentes herramientas pedagógicas que orienten a los agentes educativos, para favorecer el proceso de aprendizaje en los niños de o a 6 años de edad, como es el caso de la ciudad de Villavicencio, en donde se puede establecer que no se cuenta con algún tipo de investigación que plantee dichas herramientas.

1. Trabajo presentado en La Corporación Universitaria Minuto de Dios, a cargo de las profesoras Alexandra Giovana Cortés Santacruz, Magister en Educación y Giovanna Yalile García Cardona, Estudiantes de Maestría en Gestión de la Tecnología Educativa.

2. Lic. Pedag. Infantil, Esp, Mag. Semillero de Investigación Trillas del Conocimiento. Corporación Universitaria Minuto de Dios, Regional Llanos.

Correo electrónico: alexandra.cortes@uniminuto.edu

3. Lic. Pedag. Infantil, Esp. Semillero de Investigación Trillas del Conocimiento, Grupo Gavieros. Corporación Universitaria Minuto de Dios, Regional Llanos

Correo electrónico: gigarcia@uniminuto.edu.co 
Palabras clave: procesos pedagógicos, estudiante, educador, estrategias pedagógicas, didáctica.

\title{
Teaching strategies to enhance learning in children from 0 to 6 years at Villavicencio Colombia
}

\begin{abstract}
Education in the early years of life is a continuous and integral process, product of multiple experiences that the school provides to children, through interaction with society and the environment, being the basis of comprehensive education of the human being. One of the many tools or pedagogical strategies are play, performing arts, painting and reading, which are transformed into playful-pedagogical strategies that favor the integral development of the child. This bibliographic review aims to present the results of several researches that contain a plurality of methodological tools that teachers can use in the classroom to develop better learning in the child. There is a variety of strategies implemented by teachers in educational institutions; however, there is no research that produces and integrates the different pedagogical tools that guide the educational agents, to favor the learning process in children from o to 6 years of age, as is the case of the city of Villavicencio, where it can be established that there is not some type of research that presents these tools.
\end{abstract}

Keywords: pedagogical processes, student, educator, pedagogical strategies, didactic. 


\section{Introducción}

La infancia es una etapa fundamental en el ciclo de vida de los seres humanos, que inicia desde la gestación y se extiende hasta los cinco años de edad, en la que se establecen las bases para el desarrollo cognitivo, emocional y social del ser humano (Morrison, 2005); es por ello, que las experiencias que el niño tenga durante esta etapa serán fundamentales para su vida futura. De esta manera, las prácticas pedagógicas que se propicien en una educación inicial de calidad deben ser planeadas e intencionadas, respondiendo a las necesidades educativas de los niños (Backer, 2003); por ende, los diferentes aprendizajes que se adquieren durante este periodo dejan una huella imborrable para toda la vida.

Este artículo permitirá realizar una recopilación bibliográfica cuyo objetivo es conocer e identificar las diferentes herramientas y estrategias pedagógicas que los docentes pueden implementar en el aula de clase; detallando en qué consiste cada una y qué transcendencia tiene para el niño en su desarrollo integral.

Las estrategias pedagógicas para los niños de o a 6 años de edad, son herramientas esenciales dentro de la educación inicial, esto se debe principalmente a la transformación social, avances tecnológicos y por su puesto a los intereses propios de los estudiantes (Backer, 2003). Estos mismos factores son los que le han exigido a la educación implementar dentro de su currículo escolar, metodologías que motiven el quehacer del estudiante, dentro del aula, esto con el fin de favorecer los procesos de enseñanza y fomentar aprendizajes significativos, que apunten a potencializar el desarrollo integral de los estudiantes.

Dentro de la literatura académica se pueden encontrar varias posiciones, frente a la diversidad de estrategias metodológicas que el docente puede implementar en el aula de clase, encontrando que las herramientas pedagógicas deben apuntar a reconocer en el estudiante sus propias capacidades (Pérez, 2012); permitiéndoles así, por medio de las mismas, que el niño reconozca e identifique su vocación y que ésta le permita salir adelante en un mundo que en muchas ocasiones ignora a quienes no tienen un saber hacer; es decir, que el maestro se dedique, (por medio de las estrategias pedagógicas) a descubrir formas y técnicas, que conlleven al desarrollo humano integral de los niños. 
Dentro de esta revisión bibliográfica encontramos también a Morrison (2005), quien plantea la importancia no en la estrategia pedagógica, sino en la técnica que el maestro implementa en el aula de clase; influyendo además, factores externos como ubicación, espacio físico, tipo de material didáctico, postura del maestro y demás; es por esto, que esta autora afirma, que aún si se contara con un entorno y material excepcional, no funcionaría si el maestro no tiene la actitud correcta, generando que el estudiante nunca reciba, asimile, ni ponga en práctica el contenido expuesto por el docente.

De acuerdo a Aragón \& Jiménez (2012), la teoría de los estilos de aprendizaje debe ser incorporada en el trabajo cotidiano de todos los docentes, lo cual indica que las estrategias pedagógicas manejadas en un aula de clase han de ser acorde a los diferentes estilos de aprendizaje existentes; para que de esta manera se logre una efectividad en la enseñanza y a su vez se eleve la calidad educativa.

\section{Referentes teóricos}

Desde la antigüedad, siempre se ha notado el interés de atender la necesidad de apoyar el proceso de enseñanza a nivel mundial; por ende la importancia de distinguir entre la historia de la educación y la pedagogía. La educación aparece como acción espontánea y natural, surgiendo mucho después el carácter intencional y sistemático de la pedagogía. El surgimiento de la pedagogía en el escenario educativo, se da con el fin de refinar técnicas y métodos para transmitir un conocimiento, así como teorizar sobre los hechos educativos que se presentan en cada momento histórico (Pérez, 2012).

La evolución del ser humano ha creado enseñanzas significativas las cuales se han transmitido con el fin de seguir mejorando en la calidad educativa. El hombre por naturaleza es curioso, explorador e investigativo, lo que ha permitido tener cambios y mejoras significativas en la calidad de vida, y es en este proceso de transferencia de conocimientos a lo que hoy se le llama educación, con unas tradiciones y costumbres que identifican las diferencias de otros países, sociedades, comunidades en el mundo, en las cuales se convierten en una gama de concepciones religiosas, filosóficas y tecnológicas (Backer, 2003) que son la base de las idiosincrasias de cada país. Todo esto fusiona en la 
concepción pedagógica actual y por lo tanto es lo que le da vida y sentido de pertenencia al acto educativo.

El origen de la educación se da en la evolución del hombre, que permite mejorar su calidad de vida, se puede ver desde cuando el hombre trasciende del nomadismo al sedentarismo y esas mismas experiencias o saberes se comienzan a transmitir entre los integrantes de estas mismas familias, comunidades y las primeras ideas pedagógicas. La complejidad de la educación comienza a aparecer por la comunicación que se establece a través del intercambio de mercancías entre diferentes grupos de diversos lugares (Lanfrancesco, 2003). Por otro lado, también se originaba la división de clases sociales de forma incipiente y rudimentaria que marcaría en siglos posteriores a la educación.

De acuerdo a lo anterior, por mucho tiempo se ha venido hablando sobre estrategias en el ejercicio pedagógico, sirviendo como apoyo a esta investigación; es así, como se puede evidenciar la existencia de ellas que facilitan el aprendizaje de los contenidos aplicados al niño, y que permiten desarrollar sus capacidades y destrezas en todas las dimensiones; bajo la supervisión del docente, para hacer el aprendizaje más significativo, teniendo siempre en cuenta que en algunas de las estrategias se deben aplicar metodologías, para que su actuar sea independiente, autónomo y transforme al estudiante en un participante activo dentro de su propio proceso de aprendizaje (Moya, 2009).

La diversidad de estrategias pedagógicas además de influir en la interiorización de contenidos, cumplen con otra función, que es desarrollar habilidades motrices, cognitivas, comunicativas y estéticas, las cuales permiten un desarrollo integral en el estudiante. El docente que facilita y promueve el desarrollo de las habilidades, es el que brinda las herramientas necesarias para comprender el mundo que lo rodea(Lanfrancesco, 2003).

\section{Metodologías en el quehacer docente}

No hay un método exacto para mejorar el quehacer pedagógico del docente (Pérez, 2012), pero si se podrían definir o crear estrategias para mejorar la práctica educativa. El quehacer, debe hacerse de una manera conciente donde se facilite el aprendizaje, el desarrollo y la calificación; 
así al preparar una clase o una actividad con procesos pedagógicos, ésta debe evidenciar los resultados en el proceso evaluativo que valora la apropiación del contenido.

Es por eso que es indispensable y obligatorio conocer y aplicar las herramientas pedagógicas de una manera efectiva, dejando la forma rutinaria y tradicional (Backer, 2003); la metodología se debe dar de una manera adecuada, conociendo las temáticas, el contexto escolar y el ambiente para favorecer un mejor aprendizaje. Las herramientas pedagógicas son para el docente las estrategias que le permiten llevar y transmitir el conocimiento de los temas de una manera significativa y divertida, pero solo si se utilizan de una forma adecuada (Morrison, 2005). Para saber cuál es la forma adecuada de hacer buen uso de las herramientas pedagógicas, se debe planear la clase de una manera consiente, pensando siempre que sea innovadora, comprometida y buscando que estimule y desarrolle las habilidades del estudiante.

Algunas herramientas pedagógicas indispensables en el quehacer de los docentes innovadores en el preescolar son:

\section{El juego}

El juego es parte fundamental de la diversión, ya que es una actividad natural y necesaria en el ser humano (Tourtet, 2003). En el quehacer pedagógico, especialmente en el preescolar, se ha tomado el juego como estrategia de enseñanza - aprendizaje ya que los niños se encuentran en la edad de construcción de su personalidad y en el desarrollo de las dimensiones (García, 2013). Muchas han sido las investigaciones realizadas en torno a los beneficios del juego; según García (2013), desde la pedagogía tradicional se ha hecho un llamado a los docentes para aplicar ésta como herramienta permanente de formación que permita dinamizar y articular los contenidos y acciones en educación integral.

Existen diversos juegos, los cuales se pueden organizar por características, tipos y clases, brindando enormes beneficios al ser humano, ya sean niños, jóvenes, adultos o ancianos. Para Tourtet (2003) las estrategias más sobresalientes infieren en la motivación, la exploración, el aprendizaje y la potencialización de habilidades del pensamiento (observar, analizar, asociar, descubrir, identificar, resolución de problemas); estimulando todas las dimensiones del desarrollo en el estudiante; permitiendo expresiones artísticas heterogéneas (bailar, 
cantar, hablar, pintar, dibujar); promoviendo el ambiente escolar, fomentando el ejercicio y la interiorización de valores que estimulen la imaginación, la creatividad, la actitud lúdica frente a la vida; contribuye a la capacidad de asombro y sorpresa; a través del juego dirigido siguen reglas, se respeta el ritmo y las capacidades de cada estudiante; permite situaciones para que el niño acepte el mundo que lo rodea; construye reglas para una sana convivencia; permite aceptar diferencias, fortalezas y debilidades a través de la relación social que genera y potencia el desarrollo psicomotor y el vocabulario generando destrezas mentales y físicas (Moya, 2009).

En síntesis, es fundamental realizar el trabajo pedagógico en base a juegos didácticos, creativos, apropiados, con objetivos claros y además, que sean atrayentes para los estudiantes; puesto que "Ningún intento será en vano a la hora de querer convertir el aula de clases en un espacio para el descubrimiento y la solidaridad, el conocimiento, el respeto a las ideas y la diversidad, a la formación de ciudadanos críticos y útiles a la sociedad... Es este el anhelo de cualquier maestro con vocación..." (Sierra \& Guédez, 2006).

\section{El ambiente}

El ambiente es considerado como una herramienta que el docente puede hacer uso para promover el aprendizaje, es un factor importante al momento de llevar a cabo un proceso de enseñanza-aprendizaje, es así que los docentes deben favorecer un espacio donde el estudiante a través de la observación y el entorno natural incida de manera significativa en la labor diaria del aprendizaje, tanto el docente como el niño, utilicen dentro de su labor pedagógica, sus elecciones y la relación con los demás (Tourtet, 2003). El ambiente que el docente escoja es muy importante debido a que es el lugar donde los niños pasan la mayoría de su tiempo con sus maestros. Pedagogos como María Montessori han promovido el uso de dichos ambientes escolares, para potencializar en los niños sus habilidades de una forma espontánea y vivencial (García, 2013).

Adicionalmente, es importante tener en cuenta que las transiciones educativas se conceptualizan como momentos críticos de cambio que viven los niños y las niñas al pasar de un ambiente a otro, pero que a la vez abren oportunidades para su desarrollo humano y su aprendizaje para la vida y la escuela (Alvarado \& Suárez, 2009), por tanto para crear un 
ambiente de aprendizaje apropiado, se ha de tener en cuenta los actores, los escenarios y las prácticas educativas.

\section{Artes plásticas}

Las artes plásticas en la educación no deben tomarse solo como una materia en el plan de estudios (Pérez, 2012). El arte se debe tomar como una materia transversal o complementaria para las demás, haciendo uso de ella, se puede pensar de una manera creativa y crítica, llevando a formar conocimientos a partir de la experiencia; generando así, su propio significado del mundo. Dewey, considerado como uno de los precursores que aportó más sobre las artes plásticas, nos aporta que los pedagogos han de incluir para el buen desarrollo de las diferentes áreas curriculares las artes, ya que estas juegan un papel importante en el proceso corporal fino y motor de los niños en edad infantil.Adicional a lo anterior, el manejo de las artes en la educación, se toma como un proyecto educativo que se fundamenta más en la experiencia que en los productos finales (Tourtet, 2003).

Son muchos los materiales que se manejan en las artes plásticas, cada uno con características diferentes a los demás desde su textura, color, tamaño, diseño, aroma, entre otras. Por eso el uso de estos materiales brinda una gran ayuda en la educación (Lanfrancesco, 2003). En síntesis, se puede establecer que las artes plásticas se deben enseñar en la escuela porque permiten abrir una puerta a la exploración, a la creatividad y a la experimentación en los niños (Rollano, 2004). Jackson (1994) retoma a Dewey (1934) en su idea de que durante el proceso creativo se reflexiona a medida que se involucra en el proceso, el cual permanece como un aprendizaje significativo.

Por tanto, el arte es otra herramienta pedagógica que favorece la adquisición del aprendizaje, porque permite realizar actividades dinámicas y unificadoras como el dibujo, la pintura, el diseño y el moldeado, formando una situación compleja que permite que el niño seleccione, interprete, plasme o diseñe sus vivencias y de esta manera, el docente puede llegar a conocer un poco más del infante en cuanto a lo que siente, piensa e interpreta (Salido \& Salido, 2013). Este mismo autor en su libro Creative and Mental Growth publicado en 1947, hace un gran aporte donde presenta una descripción detallada del proceso de evolución en el lenguaje gráfico que va manifestando el niño a medida que se desarrolla física e intelectualmente. 
Los beneficios de las artes plásticas son estimular la creatividad, agilizar el cerebro, estimular los sentidos (tacto, olfato, visión, gusto), permitir un mejor desarrollo físico-motor e intelectual en la vida del niño, los niños aprenden a llevar su propio control del ritmo y creatividad, se les facilita cualquier tipo de trabajo asignado en la escuela y es una estrategia muy diversa (Rollano, 2004).

Por medio de las artes plásticas se pretende demostrar cómo lo experimental tiene relación con lo afectivo, de igual forma con la creatividad e imaginación del individuo: expresión +creatividad= autoconcepto positivo (Clemente, 2008).

Cabe resaltar, que el niño preescolar hace sus primeras incursiones en el mundo social y comienza a asistir a centros de educación, hecho este que reafirma su proceso de autonomía y de desarrollo individual, pero a la vez trae consigo momentos de tensión y ansiedad a los pequeños. La Intervención Terapéutica "Arteterapia" para niños en edad preescolar "basa su metodología en el juego y en la capacidad creativa de resignificar la realidad" (Martínez, 2009), lo cual ratifica la importancia del manejo de las artes en el quehacer pedagógico.

\section{La lectura}

Una de las grandes problemáticas que se han evidenciado en las pruebas de conocimiento escolar, tienen que ver con la lectura y su escasa comprensión; esto debido quizás, al crecimiento tecnológico, que a pesar de que son herramientas educativas, en su gran mayoría son utilizadas como fuentes de entretenimiento y no como apoyo pedagógico dentro y fuera del aula (Gassó, 2005), identificándose que el amor por la literatura y la lectura se va perdiendo en la medida que los programas de televisión aumentan. Para esto se han realizado diversos estudios que han dado como resultado que, si se incentiva y promueve la conciencia lectora desde niños, muy seguramente se cambiará el panorama actual (Solana, 2006).

Durante la edad preescolar es necesario que el niño reciba la estimulación necesaria para un buen desarrollo de la literatura, por ende no se trata de una serie de actividades meramente mecánicas: se trata de actividades que deben portar un sentido para la actividad del niño a fin de promover su aprendizaje (Rabazo \& Moreno, 2007). Al respecto, Gonzáles y Delgado (2009) afirman que los niños preescolares 
que reciben entrenamiento en lenguaje escrito tienen mejor desarrollo de lenguaje oral, lo cual facilita su proceso lecto-escritor dentro del establecimiento educativo.

En América Latina y Europa en el año 2001, se implementó un proyecto llamado la Bebeteca, el cual buscaba que los padres motivaran a sus hijos a leer, como parte fundamental de su proceso lector; lo cual ayudaría a manejar procesos que aumenten su parte lingüística. Por lo anterior, en Colombia, después de los buenos resultados obtenidos por el taller de espanta pájaros; se implementó un proyecto cultural pedagógico dirigido a la primera infancia, que anima a la lectura y la expresión artística, propiciando un encuentro creativo con la literatura y el arte. Según la psicóloga Yolanda Reyes, una de las promotoras del proyecto La Bebeteca, se busca que los niños lleguen a una temprana edad a conocer la lectura, porque así será más fácil aplicarla en su vida, sin necesidad de llegar a ella obligados por una tarea. La importancia de la lectura a temprana edad, radica en la habilidad de despertar la imaginación, traslado al niño(a) a mundos que no conoce; se amplía el vocabulario, mientras se lee se conocen nuevas palabras; se disminuyen las faltas de ortografía, cuando se lee y se repiten muchas veces, las palabras se graban en la memoria; permite portar nuevos conocimientos, es una gran compañía en los momentos de soledad; ayuda a gozar de una buena salud mental, aleja el aburrimiento, brindando diversión. A los niños les gusta repetir las mismas lecturas, según Ibañez (2006).

Dentro del desarrollo del proceso lector, se encuentran inmersos niños y niñas menores de siete años que son llevados a la búsqueda de la lectura mediante estrategias didácticas utilizadas en el aula de clase por los docentes para incentivarlos al magnífico mundo de la lectura, buscando no generar para ellos una obligación, sino por el contrario una dinámica activa de conocimiento, orientados por la motivación, alegría, interés y amor hacia cada uno de los libros (Holguín, Sierra \& Quiñones, 2012).

Es elemental dentro del proceso de enseñanza aprendizaje, fijar en el niño el hábito de la lectura como tal, y a su vez determinar aquellas posibles causas que propician o desmotivan al niño(a) al deseo de aprender a leer dentro de su mundo, su propio hogar, en su entorno y en preescolar (Castellanos, Lozano \& Osorio, 2015).

Como complemento a los aportes antes mencionados, es de gran importancia que el docente se apropie del conocimiento fonológico 
(sílaba, principio-rima, fonema) y el conocimiento de las letras en la predicción del rendimiento temprano en lectura y escritura. Todos los niños son pre lectores en el momento de la primera medida (Fundación Infancia y Aprendizaje, 2007).

Debido a la gran importancia que tiene el desarrollar un buen proceso lector con el estudiante, se ha venido desarrollando material educativo multimedia basado en el método de lectura Doman, para facilitar la enseñanza de la lectura desde el nivel preescolar (Ángeles, Gómez, \& García, 2013).

En términos generales, un docente que busque desarrollar habilidades lingüísticas en sus estudiantes, debe plantear y diseñar estrategias para lograr que los niños desarrollen destrezas para escribir de una manera eficaz (Atorresi \& Bengochea, 2010). Es indispensable tener siempre presente que la familia es fundamental en este proceso, ya que el infante observa y aprende el significado de la lengua escrita y si se promueve la lectura en este núcleo, el niño mostrará mayor interés y motivación para aprender a leer (Figueres \& Pujol, 2006). Por tanto, familia, escuela y tecnología han de ir de la mano en este proceso lector del estudiante.

\section{La tecnología}

No se podría hablar de motivaciones, intereses y amor por la lectura, sin dejar a un lado los avances tecnológicos; son éstos que en todo el mundo hacen que sea necesaria la implementación y el uso y más aún dentro de la escuela como parte cotidiana para la mayoría de las personas. Muchos pensaran que los niños(as), en el nivel preescolar, son muy pequeños para entender la tecnología; pero la verdad es que son ellos mismos quienes les enseñan muchas veces a los adultos. Desde esta perspectiva, es necesario que en la escuela los niños(as) tengan la posibilidad de relacionarse con la tecnología, estando estas prácticas enfocadas al buen uso de ellas, a través de las herramientas pedagógicas que brindan y que igualmente sean apropiadas al contexto y a la edad en que el niño(a) se desarrolle (Gervilla, 2006).

El uso de la computadora enriquece el lenguaje de los niños como cualquier centro educativo tradicional siendo esta una herramienta de enseñanza, pero su uso se debe hacerse con responsabilidad, de manera reflexiva, con una buena y adecuada preparación para que no 
se desvíen del arte pedagógico que ofrece este (Ramírez \& Burgos, 2010).

\section{Los Materiales}

Los materiales didácticos son un elemento fundamental, en cuanto al uso de las herramientas pedagógicas, estos provocan la actividad y construcción de conocimiento en el niño, ofrecen una idea del tipo de trabajo que se llevará a cabo en el aula (Sanchidran \& Ruiz, 2010).

Diferentes objetivos, motivar diversas actividades y fomentar la creatividad, entre otros. Los materiales comerciales, los recuperables del medio, o los traídos de la casa, tienen implícitos valores y una determinada idea metodológica. El uso de algunos de ellos, como embaces, cajas o botellas de plástico, cartón, semillas, piedras, hojas, palillos, entre otros, fomentan la creatividad y favorecen las herramientas pedagógicas que ayudan a los docentes a buscar nuevas opciones que mejoren el aprendizaje (Sugranes \& Monserrat, 2012).

Es importante ofrecer a los niños materiales que beneficien y potencien su desarrollo cognitivo, que permitan la resolución de problemas y estimulen la memorización, agilidad mental, entre otras. Como ya lo exponemos anteriormente, no se necesita de materiales comprados en el extranjero ni de las últimas innovaciones, sólo basta con recreary adecuar el espacio para que sea el mismo niño que motivado utilice todos los recursos que tiene a su alrededor pero que servirán para potenciar su aprendizaje.

\section{Las rondas}

Otra de las herramientas pedagógicas olvidadas en algunos casos, es la Ronda, que facilita el aprendizaje del niño en una forma creativa y divertida. Es un elemento de expresión ritmo-plástica muy completa, que permite la participación activa del niño en forma espontánea y va mejorando su formación integral como tal; además, estimula el desarrollo social, desarrolla la creatividad, imaginación y contribuye a la solución de problemas (adaptación, ubicación, respeto al compañero, manejo de espacio) (Requena \& Sainz, 2010). Esta herramienta es un medio en el que el niño desarrolla la coordinación y ayuda en la formación de su lenguaje, su libre expresión, se adquieren hábitos, valores, reglas, motivando así la 
participación, la creatividad, la imaginación y el deseo por descubrir nuevas cosas.

\section{La música}

La música dentro de las artes es un medio de expresión y comunicación en la que intervienen el tiempo, los sonidos, el ritmo y el movimiento. Esta hace parte de la vida y el ser humano se convierte en receptores de cualquier información sonora, como por ejemplo: los sonidos de los coches, la radio o televisión, etc. Solana (2006), indica que a través de la expresión musical, se logra la motivación de los alumnos y, además, de ayudar en su formación integral, tanto en el aspecto cognitivo, afectivo, motor, también lo hace en el aspecto social. De igual forma, dicho autor indica que la música además de ser divertida, mejora el desarrollo cerebral y, aún más, mejora las habilidades en la lectura y las matemáticas.

La educación musical como aporte esencial, en la formación integral del niño, contribuye en gran parte al desarrollo de habilidades y valores que afianzan su autonomía. Al plantearse las diferentes estrategias para guiar el proceso musical del estudiante, se ha propuesto la articulación de las demás artes en este proceso como lo son danza, pintura, teatro (Capacho \& Dura, 2006), con ellos el niño disfruta y activa sus emociones lo que permite que sea alegre, creativo y que a través de ello su aprendizaje sea significativo.

\section{LosTíteres}

El títere brinda al niño una oportunidad para crear con su mente y su mano, ejercitando el movimiento independiente de los dedos; es apropiado para llevar al niño a un conocimiento, ayuda a vencer la timidez, contribuye al desarrollo del lenguaje, y ayuda a expresar sentidos y emociones (Sugranes \& Monserrat, 2012). Hay muchas clases de títeres que pueden adquirirse en el comercio o construirse por el mismo estudiante, siendo esto último lo más recomendable, ya que se logra mayor apropiación del títere por parte de niño(a) y por lo tanto tendrá mayor confianza para expresar lo que desee.

Maya (2005) expresa que existe una considerable cantidad de objetivos que pueden lograrse con el "taller educativo", del cual se resalta el que 
dichos talleres promueven y facilitan una educación integral y simultánea en el proceso de aprendizaje en cuanto al aprender a aprender, aprender a hacer $y$ el aprender a ser. Por medio de los títeres los niños fantasean, recrean sus propias historias y toman confianza en ellos mismos; hay un detalle importante es que se muestran tal y como son y dejan al desnudo su inocencia al igual que el contexto y ambiente familiar que les rodea.

\section{La Danza}

La danza está formada por los elementos de movimiento corporal, ritmo, música y expresión o comunicación. Se puede definir de diversas formas, según el punto de vista que se adopte. Según Solana (2006) de una manera amplia, se puede decir que la danza es un arte visual que se desarrolla en el tiempo y en el espacio y se asocia a la música e incluso a la palabra. La práctica de la danza permite al niño(a) adquisición y desarrollo de las habilidades, de su motricidad gruesa, destrezas, favorece su coordinación, fortalece su lateralidad, atención, creatividad, memoria. Por medio de la danza, el niño puede expresar sus sentimientos, emociones e interactuar con los demás. La danza por sí misma juega ya un papel muy importante en el terreno educativo. Su valor es real en la medida que despierta en el estudiante su propio encanto y lo que de ello resulta: lo torna visible al momento de bailar y poner en práctica su cuerpo con movimientos cadenciosos al ritmo de una pieza musical. Muchas de las actividades que realizan los niños en sus juegos tienen relación con las manifestaciones artísticas; al cantar, pintar, bailar, actuar y representar situaciones diversas, el niño disfruta y expresa sus sentimientos y emociones, creando ambientes favorables que le ayudan a percibir su entorno de manera más profunda y natural (Gervilla, 2006).

Además de lo citado anteriormente, cabe resaltar que la danza "Sirve para comunicar", la mayoría de las danzas tienen una intención comunicativa, desde las danzas pantomímicas o de caracterización, pasando por el ballet clásico, hasta las que procuran la expresión de una emoción personal o una exuberancia física (Fuentes, 2006).

\section{El canto}

El canto se considera como herramienta que permite al niño ser creativo, es una actividad que contribuye de manera especial al desarrollo 
de la inteligencia de niños y adolescentes, genera alegría y facilita la comunicación y el intercambio de experiencias, conocimientos y promueve la apertura natural y sincera entre los seres humanos (Salido \& Salido, 2013).

Las canciones pueden formar parte de la vida, puesto que existen un sin número de temas que se pueden usar en todo momento e inclusive inventar nuevas canciones; es por ello, que en las instituciones de educación universitaria, de donde egresan los formadores, se está olvidando frecuentemente la educación por el arte, en especialmente por el canto (Solana, 2006).

\section{Conclusiones}

Para concluir este artículo, es importante resaltar que todos los autores guardan estrecha relación en sus saberes, debido a la importancia que les dan a las actividades que ayudan a enriquecer los procesos de aprendizaje de los estudiantes. La variedad de didácticas sugeridas por ellos ayuda a fortalecer aprendizajes significativos permitiéndole al estudiante la interacción del objeto con su saber.

Se puede inferir que en la pluralidad de documentación revisada, se encuentra un repertorio amplio en cada una de las temáticas trabajadas; permitiendo así, a las autoras, hacer un análisis exhaustivo de lo expuesto por cada uno de los precursores investigados. Por consiguiente, se hace necesario que dentro de la documentación que se le brinde al lector, se incorporen los procesos pedagógicos que deben ser utilizados a la hora de impartir la educabilidad a los niños; teniendo en cuenta sus contextos, repertorios básicos y la edad. No se puede dejar de lado resaltar que los procesos metacognitivos trabajan de manera paralela a otros procesos cognoscitivos, como memoria, atención, lenguaje, y a su vez están relacionados con diversas áreas del conocimiento, como matemáticas, lectura, escritura y solución de problemas (Ochoa, Aragón, Correa, \& Mosquera, 2008), por ende resulta fundamental tenerlo en cuenta al desarrollar el ejercicio pedagógico.

Con el fin de obtener un excelente resultado al momento de enseñar, se deben conocer e incorporar los procesos pedagógicos básicos que permitirán que el educador haga claridad de la importancia que es utilizar 
el paso a paso adecuado a la hora del trabajo en el aula, dejando de un lado la arbitrariedad que normalmente se utiliza al interior de las clases. Se requiere que el proceso enseñanza aprendizaje se adapte a cada estudiante, en la medida de lo posible respetando sus propios ritmos y para lograrlo, los docentes deben adquirir una competencia didáctica, entendida como la capacidad de enseñar a otro a aprender de por vida (Frade, 2007). Esto ayudará que el estudiante adquiera un aprendizaje significativo y provechoso que le facilitará su vida diaria y académica.

En este orden de ideas, la educación se encuentra frente a un gran reto, consistente en una revolución educativa dirigida a crear nuevos modelos pedagógicos que respaldan una enseñanza desarrollante, orientada a fomentar la capacidad creativa de los estudiantes en todos los niveles, desde el preescolar hasta la educación superior. Se exponen los componentes del proceso educativo relevantes para el desarrollo de la capacidad creativa: actitud creativa del docente, creación y utilización de estrategias pedagógicas y didácticas en las aulas de clase, fomento de las atmósferas creativas y la emergencia de la creatividad como un valor cultural (Klimenko, 2008).

\section{Agradecimientos}

Las autoras agradecen a la Corporación Universitaria Minuto de Dios, Vicerrectoría Regional Llanos y al coordinador del Programa de Pedagogía infantil, el apoyo a la realización de esta investigación. De igual manera agradecimientos especiales a las estudiantes Mónica Gélvez, Yina Mileidy Silva y Nini Johana Galeano por sus aportes en el desarrollo y construcción de este artículo. 


\section{Referencias bibliográficas}

Ángeles, M., Gómez, M. \& García, I. (2013). Diseño de un recurso educativo multimedia basado en la Metodología Doman para mejorar la enseñanza de la lectura en el nivel preescolar. Revista DIM. № 27. Disponible en: http://dim.pangea.org/revistaDIM27/ docs/AR27lecturapreescolarMAangeles2.pdf

Aragón, M., \& Jiménez, M. (2012). Diagnóstico de los estilos de aprendizaje en los estudiantes: Estrategia docente para elevar la calidad educativa. CPU-E, Revista de Investigación Educativa, 0(9), 23-43. Disponible en: http://revistas.uv.mx/index.php/ cpue/article/view/73/148

Atorresi, A., Bengochea, R., Bogoya, D., Burga, A., Castro, M., García, F., Jurado, F. Martínez, R. \& Pardo, C. (2010). Escritura. Un estudio de las habilidades de los estudiantes de América latina y el Caribe. Santiago: Unesco-Orealc.

Alvarado \& Suárez. (2009). Formación en valores y ciudadanía: Una perspectiva desde la socialización política. En: Rodríguez, G. I. (2009). Formación en valores y ciudadanía desde una perspectiva cotidiana. (pp. 14-27). Bogotá: Idie-Editorial Delfín.

Backer, B. (2003). Actividades didácticas para la etapa preescolar. Andalucía:. Editorial Edic / Ceac.

Capacho, Y. \& Durán, Z. (2006). Diseño de talleres para la enseñanza musical en el grado preescolar. El Artista, 3,161-169. Disponible en: http://www.redalyc.org/articulo. oa?id=87400312

Castellano, Y. Lozano, T. \& Osorio, M. (2015). La literatura infantil como estrategia pedagógica para generar aprendizajes significativos en los procesos de lectura. Trabajo de grado. Ibagué: Universidad del Tolima.

Figueres, C. y Pujol, M. (2006). Propuestas de aprendizaje, leer y escribir en edades tempranas. Barcelona, CEAC.

Frade, L. (2007). Desarrollo de competencias en educación: Desde prescolar hasta bachillerato. México: Inteligencia Educativa

Franco, C. (2008). Relajación creativa, creatividad motriz y autoconcepto en una muestra de niños de educación infantil. Electronic Journal of Research in Educational Psychology, 6 29-50. Disponible en: http://www.redalyc.org/articulo. oa?id=293121940003

Fuentes, A. (2006). El valor pedagógico de la danza. Tesis doctoral, Valencia: Universidad de Valencia.

Fundación Infancia y Apredizaje. (2007). Infancia y Aprendizaje: Journal for the Study of Education and Development. Disponible en: https://dialnet.unirioja.es/ revista/702/A/2007 
García, R. (2013). Enseñar y aprender en Educación Infantil a través de proyectos. Santander: Editorial Universidad Cantiabra.

Gassò, A. (2005). La Educación infantil: métodos, técnicas y organización. Barcelona: Editorial Ceac.

Gervilla, A. (2006). Didáctica básica de la educación infantil: Conocer y comprender a los más pequeños. Madrid: Editorial Narcea.

González, M. \& Delgado, M. (2009). Rendimiento académico y enseñanza-aprendizaje de la lectoescritura en Educación Infantil y Primaria: un estudio longitudinal. Infancia y Aprendizaje, 32(3), 265- 276.

Holguín, K., Sierra, G. \& Quiñones, S. (2012). Estrategias metodológicas empleadas por los docentes de educación preescolar del municipio de San Sebastián de Mariquita (Tolima) en la enseñanza de la lectura. Revista Logos Ciencia \& Tecnología Vol 3(2). DOI: http://dx.doi.org/10.22335/rlct.v3i2.151

Ibáñez, C. (2006). Proyecto de educación infantil y su práctica en el aula. Madrid: Editorial La Muralla.

Jiménez Mendoza, W., Soto Carrión, C., \& Urrutia Huamán, R. (2015). Relación entre modelo PIHEM y gestión educativa en instituciones educativas del nivel inicial en el distrito de Abancay Apurímac Perú, 2014. Revista De Investigaciones UNAD, 14(1), 81 95. doi:http://dx.doi.org/10.22490/25391887.1347

Klimenko, O. (2008). La creatividad como un desafío para la Educación del siglo XXI. Educación y Educadores. Vol. 11(2). Disponible en: http://educacionyeducadores. unisabana.edu.co/index.php/eye/article/view/740/1717

Lanfrancesco, G. (2003). La educación integral en el preescolar: Propuesta pedagógica. Bogotá, D.C: Editorial Magisterio.

Lurán Rivero, A., Buenahora Tobar, M., \& Vargas Granados, C. (2015). Perspectiva del aprendizaje tradicional y del aprendizaje con comunicación bidireccional en actividades educativas. Revista De Investigaciones UNAD, 14(1), 253-262. doi:http://dx.doi. org/10.22490/25391887.1468

Martínez, S. (2009). Arteterapia con niños en edad preescolar. Arteterapia. Papeles de Arteterapia y Educación Artística para la Inclusión Social. Vol. 4, 159 - 175. Disponible en: http://revistas.ucm.es/index.php/ARTE/article/view/9637

Maya, A. (2005). El taller educativo. Lima: Universo S.A.

Morrison, G. (2005). Educación infantil. Madrid: Editorial Pearson.

Moya, E. (2009). Programación didáctica para educación infantil. Sevilla: Editorial Cultiva libros. 
Ochoa, S., Aragón, L., Correa, M., \& Mosquera, S. (2008). Funcionamiento metacognitivo en niños escolares en la escritura de un texto narrativo antes y después de una pauta de corrección conjunta. , 11 (2), 77-88. Acta Colombiana de Psicología.

Pérez, S. (2012). Didáctica de la Expresión Musical en Educación Infantil. Valencia: Editorial Psylicom.

Rabazo, M. J., \& Moreno, J.M. (2007). Teoría de la mente: la construcción de la mente mediante los cuentos de hadas. Enseñanza e Investigación en Psicología, 12 (1), 179201.

Ramírez, M. \& Burgos, J. (2010). Recursos Educativos Abiertos en Ambientes Enriquecidos con Tecnología. Madrid: Editorial Rústica.

Requena, D. \& Sainz, P. (2010). Didáctica de la educación infantil. México: Editorial Editex.

Rollano, D. (2004). Educación plástica y artística en educación infantil: desarrollo de la creatividad: Métodos y estrategias. Madrid: Editorial Ideas Propias.

Salido, E. \& Salido, M. (2013). Materiales didácticos para educación infantil: Cómo construirlos y cómo trabajar con ellos en el aula. Madrid: Editorial Narcea.

Sanchidran, C. \& Ruiz, J. (2010). Historia y perspectiva actual de la educación infantil. Barcelona: Editorial Graó.

Sierra, D. \& Guédez, C. (2006). Juego y aprendo a calcular. Caracas: Fe y Alegría.

Silva Carreño, W., \& Beltrán Martin, J. (2015). El rol de género como fundamento humanista de la formación para la ciudadanía. Revista De Investigaciones UNAD, 14(1), 7 - 17. doi:http://dx.doi.org/10.22490/25391887.1343

Solana, F. (2006). Educación: visiones y revisiones. México: Editorial Siglo XXI.

Sugrañes, E. \& Monserrat, N. \& Casal, S. \& Castrillo, C. \& Medina, N. \& Yuste, M. (2012). Observar para interpretar: Actividades de vida cotidiana para la educación. Barcelona: Editorial Graos.

Tourtet, L. (2003). Lenguaje y pensamiento escolar. Madrid: Editorial Narcea. 